\title{
NRQCD results on the MILC extra coarse ensemble
}

\section{Ian Allison*, Christine Davies}

Department of Physics and Astronomy

University of Glasgow,

G12, $8 Q Q$

$U K$

E-mail: i.allisonephysics.gla.ac.uk

\section{Alan Gray}

Edinburgh Parallel Computing Center

University of Edinburgh,

EH9, 3JZ

$U K$

We present preliminary results using NRQCD to describe heavy quarks on the MILC $2+1$ flavour dynamical extra coarse ensemble. We calculate the spectra of low lying states in bottomonium to complement earlier results on the finer MILC ensembles. We then exploit the coarseness of the lattices to calculate the charm propagators using NRQCD. These are used to examine the charmonium spectrum and to calclate the mass of the $B_{c}$ using NRQCD. Finally we look breifly at the $B_{d}$ and $B_{s}$ systems using the imporoved staggered formalism to describe the light valence quarks.

XXXIIIrd International Symposium on Lattice Field Theory

25th-30th July 2005

School of Mathematics, Trinity College, Dublin, Ireland

\footnotetext{
* Speaker.
} 


\section{Introduction}

Heavy quark physics presents the lattice with an invaluable opportunity to provide both theoretical insight and the key to some precise results from experiment. The use of effective field theory techniques such as NRQCD [1] allow heavy quarks to be described on the lattice avoiding the large discretisation errors of relativistic formulations.

The basic idea of these techniques is that simulations should be performed on lattices coarse enough that the cutoff $\pi / a$ separates the heavy quark mass ${ }^{1}$ scale $M$ from the dynamical scales $M v, M v^{2}$, where $v$ is the heavy quark velocity inside the bound state. This allows a non-relativistic formulation of QCD to simulate only the dynamical scales important to heavy mesons, with the physics at the heavy quark mass scale (and above) being encoded into matching coefficients.

The other key component of the calculations presented here is the use of $2+1$ flavour dynamical configurations provided by the MILC collaboration [2]. These configurations employ the $a^{2} \mathrm{im}$ proved "AsqTad" improved staggered quark action and a highly improved gluon action to describe the vacuum. Our calculations look at the coarsest ensemble generated which is comprised of around $60016^{3} \times 48$ lattices with spacing $a \sim 0.17 \mathrm{fm}$, intended to complement the coarse $(a \sim 0.12 \mathrm{fm})$ and fine $(a \sim 0.09 \mathrm{fm})$ ensembles on which the majority of results have been extracted. Part of the motivation for our calculations was to determine the feasibility of the extra-coarse ensemble for extraction of physical results in the presence of possible large discretisation errors.

In section 2 we outline the formulation used for our calculations, then in section 3 we look at the $\Upsilon$ and $\psi$ spectra. In section 4 we calculate the mass of the $B_{c}$ meson using NRQCD for both the $b$ and $c$ quarks. Finally, in section 5 we look at the $B_{s}$ and $B_{d}$ mesons by combining an NRQCD $b$ quark with an improved staggered light quark.

\section{Formulation and Technical Details}

A potential model calculation using a logarithmic potential [3] can be used to extract the typical velocity of the heavy quarks in their bound states. Doing so gives $v_{b}^{2} \sim 0.1$ for $b \bar{b}, v_{c}^{2}=0.25$ for $c \bar{c}$ and $v_{b}^{2} \sim 0.04, v_{c}^{2} \sim 0.4$ for the $B_{c}$ meson. These values justify a non-relativistic description, with the possible exception of the $c$ quark of the $B_{c}$ system. This work makes use of the non relativistic formulation of QCD (NRQCD) developed in [1]. In this formulation, the operators of the heavy quark hamiltonion are classified according to a power counting in the velocity of the heavy quark in the bound states; $v$. We use the action given in [1] which is correct through $\mathscr{O}\left(v^{4}\right)$.

We fit the mesonic corellators using the constrained curve fitting method of [ []. This method employs Bayesian techniques which utilise prior information to guide the fit, allowing all of the excited states present in the initial few time slices of correlator to be fitted. This improves the statistics of the fits while reducing the sensitivity to the number of exponentials used.

\section{The $\Upsilon$ and $\psi$ heavy quarkonium systems}

The majority of calculations using NRQCD describe $b$ quark systems, see for example [巨]]. In the quantity $a M_{Q}, M_{Q}$ for the $b \bar{b}$ is a relatively large number $\left(M_{Q} \gg 1\right)$, so that even with present

\footnotetext{
${ }^{1}$ Throughout this work, the term heavy quark refers to either the $b$ or $c$ quark.
} 
day lattices $a M_{q}$ is quite large. On the same lattices, the $c \bar{c}$ system requires an $M_{Q}$ of less that 1.0 and inverse powers of $M_{Q}$ in the NRQCD evolution equation then cause numerical instability, preventing results from being extracted. The advantage of the extra coarse configurations is that $M_{Q}=1.0$ produces mesons whose kinetic mass is close to that of the $J / \psi$ particle while the evolution equation remains stable.

In NRQCD, the zero of energy is shifted, so that the energy of a meson with momentum $\mathbf{p}$ is

$$
E(p)=E(0)+\sqrt{p^{2}+M^{2}}-M,
$$

which allows us to extract a physical mass using states at non-zero spatial momentum. This kinetic mass is commonly used to tune the input quark mass. From figures 1 and 2 it is clear that the $b$ quark mass $\left(M_{b}=4.0\right)$ is accurately tuned, while the $c$ quark mass $\left(M_{c}=1.0\right)$ is about $12 \%$ too low.

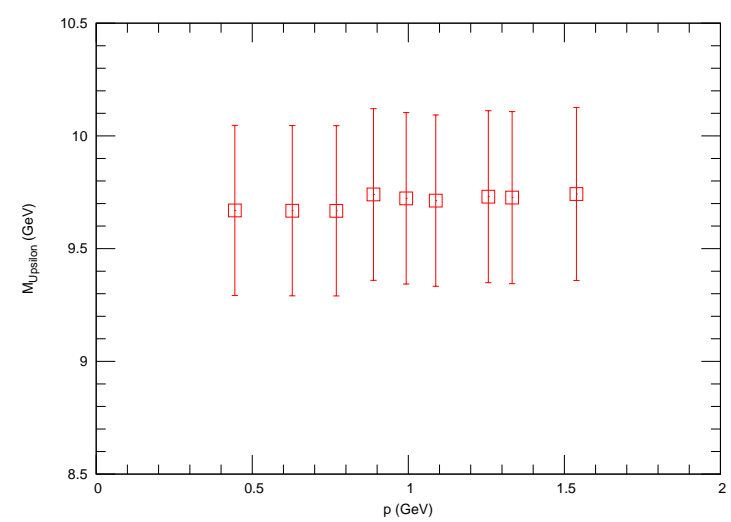

Figure 1: Kinetic Mass of the $\Upsilon$

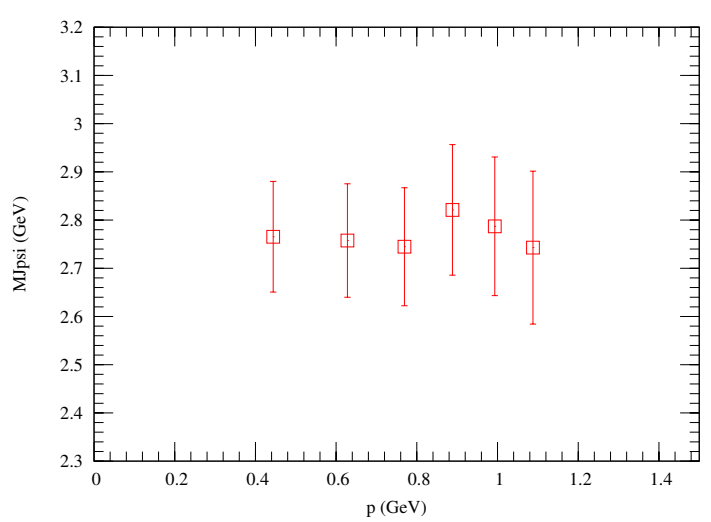

Figure 2: Kinetic Mass of the $J / \psi$

Rearranging expression (3.1) it is possible to extract the effective speed of light; $c^{2}=\left((\Delta E)^{2}+\right.$ $2 M \Delta E) / p^{2}$, where $M$ is the kinetic mass determined at some fixed $\mathbf{p}$ (taken to be $\mathbf{p}=1$ in this work). Of course, this quantity should be equal to 1 , but on the lattice discretisation effects can ruin this. We found $c^{2}=1$ to within statistical errors of $1 \%$ and $4 \%$ for the $b \bar{b}$ and $c \bar{c}$ systems respectively. This compares very favourably with relativistic formulations without high levels of improvement, where deviations of up to $10 \%$ are not uncommon [6].

The systematic way in which NRQCD was developed makes it possible to estimate the effect of higher order terms left out of the simulation hamiltonian. To do this the expectation values of these operators are calculated using potential model estimates of $\left\langle\mathbf{p}^{2}\right\rangle$ and $\left\langle\mathbf{p}^{\mathbf{4}}\right\rangle$. These corrections fall naturally into three categories.

Relativistic corrections: The main relativistic correction comes from the first term left out of the relativistic expansion of the energy momentum relation ${ }^{2},\langle\mathbf{p}\rangle^{6} / 8 M_{Q}^{5}$.

Radiative corrections: The effective field theory coefficients $c_{i}$ of the terms appearing in the NRQCD hamiltonian take the form $c_{i}\left(M_{Q}, \alpha_{s}\right)=1+c_{i}^{(1)}\left(M_{Q}\right) \alpha_{s}+\cdots$, where the $c_{i}^{(1)}$ are calculable in perturbation theory but are expected to be $\mathscr{O}(1)$. We estimate the effect of radiative corrections for the kinetic term; $\alpha_{s}\left\langle\mathbf{p}^{4}\right\rangle / 4\left(M_{Q}\right)^{3}$, and for the Darwin term ${ }^{3} ; 4 \pi \alpha_{s}^{2} \psi^{2}(0)^{2} /\left(3 M_{Q}\right)^{2}$.

\footnotetext{
${ }^{2}$ The correction terms all have an extra factor of 2 included because of the two quarks in the meson.

${ }^{3}$ This expression comes from estimating the $\Delta . E$ term in a potential model.
} 
Discretisation corrections: The terms in the heavy quark hamiltonian which correct for discretisation errors in the lattice spatial derivative and temporal derivative have corrections of the form $\alpha_{s} a\left\langle\mathbf{p}^{4}\right\rangle / 8 n\left(M_{Q}\right)^{2}$ and $\alpha_{s} a^{2}\left\langle p_{i}^{4}\right\rangle / 12\left(M_{Q}\right)$ respectively.

In this work we are interested only in energy splittings, so that adding errors from these sources for each state in quadrature is an over estimate. Instead, we calculate the above corrections using potential model estimates of the difference in the expectation values of the various splittings. The values calculated are shown in table 1. It can be seen from this table that the errors are substantially less for the radial splittings than for the orbital splittings. This is just a statement of the similarity of the dynamics of the radial states leads to a more efficient cancellation of higher order effects.

\begin{tabular}{c|c|c|c|c} 
Correction & $\Upsilon(2 S-1 S)$ & $\psi(2 S-1 S)$ & $\Upsilon(1 P-1 S)$ & $\psi(1 P-1 S)$ \\
\hline$\alpha_{S} \delta p^{4} / 4\left(M_{Q}\right)^{3}$ & 0.55 & 1.07 & 2.19 & 1.73 \\
\hline$\delta p^{6} /\left(M_{Q}\right)^{5}$ & 0.30 & 1.64 & 0.92 & 1.47 \\
\hline $4 \pi \alpha_{s}^{2} \psi^{2}(0) / 3 M^{2}$ & 0.62 & 0.90 & 1.79 & 2.82 \\
\hline Total Rel./Rad. & $\mathbf{0 . 8 8}$ & $\mathbf{2 . 1 6}$ & $\mathbf{2 . 9 7}$ & $\mathbf{3 . 6 2}$ \\
\hline \hline$\alpha_{S} a^{2} \delta p_{i}^{4} / 12 M_{Q}$ & 2.2 & 0.61 & 8.76 & 0.99 \\
\hline$\alpha_{S} a \delta p^{4} / 8 M^{2} n$ & 0.61 & 0.23 & 2.45 & 0.37 \\
\hline $4 \pi \alpha_{s} a \psi^{2}(0) / 15$ & 2.47 & 0.51 & 7.17 & 1.60 \\
\hline Total Disc. & $\mathbf{3 . 3 6}$ & $\mathbf{0 . 8 3}$ & $\mathbf{1 1 . 5 8}$ & $\mathbf{1 . 9 2}$ \\
\hline \hline Total & $\mathbf{3 . 4 7}$ & $\mathbf{2 . 3 1}$ & $\mathbf{1 1 . 9 6}$ & $\mathbf{4 . 0 9}$
\end{tabular}

Table 1: Systematic corrections to heavy quarkonium splittings as a percentage of the experimental splitting

From these determinations it is clear that the $2 S-1 S$ splitting of the $\Upsilon$ system presents the best opportunity for extracting the lattice spacing for $b \bar{b}$ states, it gives $a^{-1}=1.121(62)(31)$ where the first error is statistical and the second comes from the above systematic considerations. Increased statistical precision means that $\psi(1 P-1 S)$ splitting the best choice for setting $a$ from the $c \bar{c}$ system, giving $a^{-1}=1.111(29)(23)$. The agreement of these two determinations is unsurprising when using dynamical gauge configurations.

In figures 3 and $\$$ we plot the spectra of $b \bar{b}$ and $c \bar{c}$ systems respectively. The agreement of the lattice points with the experimental values (shown as lines) is good in the $\Upsilon$ system. In the $\psi$ system, there is a noticeable discrepancy in the hyperfine splitting of $44 \mathrm{MeV}$. This is too large to be fully explained by the systematic corrections of table 1 but is at least in part due to the fact that the $c$ quark mass was tuned too low.

\section{The $B_{c}$ Mass}

Last year we presented a calculation of the mass of the $B_{c}$ meson using NRQCD $b$ quarks and Fermilab method $c$ quarks [7]. The use of dynamical configurations in this calculation allowed a level of precision which, when combined with the experimental status of the $B_{c}$ mass at that point, allowed us to call our calculation a prediction. We found $M_{B_{c}}=6.304(4)(11)\left({ }_{-0}^{+18}\right)$ [8], where, in order, these errors were due to statistics and chiral extrapolation, uncertainty in setting the input quark masses, and an estimate of higher order corrections to Fermilab quark action. 


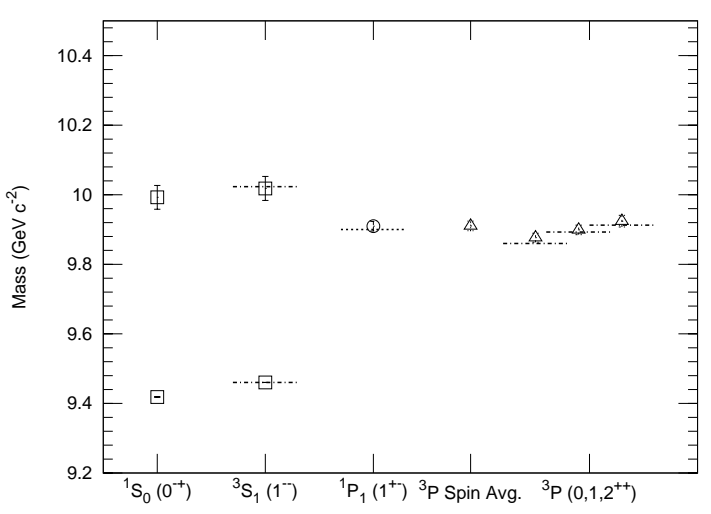

Figure 3: The $\Upsilon$ spectrum. $\Psi(1 P-1 S)$ has been used to set the lattice spacing and the absolute scale has been set by the ${ }^{3} S_{1}$

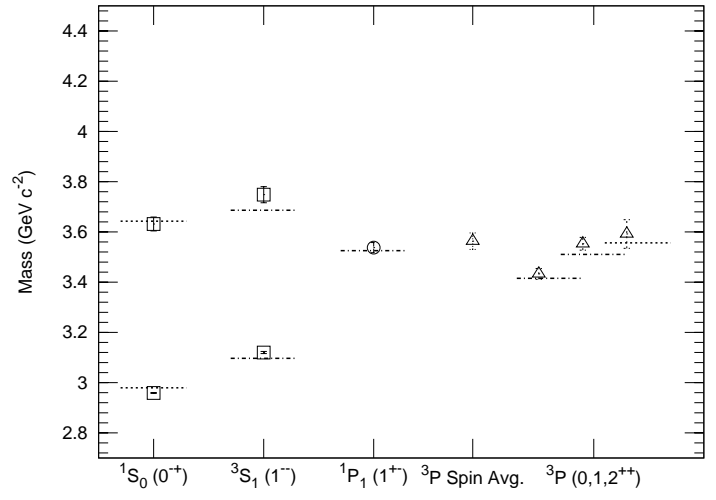

Figure 4: The $\psi$ spectrum. The $\Psi(1 P-1 S)$ has been used to set the lattice spacing and the absolute scale has been set at the spin average of the $1 S$ states

Soon after our result was published, new determinations of the $B_{c}$ mass were made at the Tevatron [9]. They quoted a preliminary result of $M_{B_{c}}=6.2879(48)(11)$ where the errors were statistical and systematic respectively.

Our method relies on forming the difference,

$$
m_{B_{c}}-1 / 2\left(m_{\Upsilon}+m_{J / \psi \eta_{c}}\right)=M_{B_{c}}-1 / 2\left(M_{\Upsilon}+M_{J / \psi \eta_{c}}\right)
$$

where the quantities on the left are lattice energies, and those on the right are physical masses. The states labelled $\psi \eta_{c}$ are the spin average of the $\psi$ and $\eta_{c}$. Repeating our earlier calculation on the extra coarse ensemble but using NRQCD to describe both valence quarks, we find 6.274(1)(25), where the first error is statistical, and the second is calculated in the same way as those in table 1 . Encouragingly, this value is in agreement with both the experimental value and our previous result.

\section{Heavy light results}

Finally, we also looked at the $B_{s}$, and $B_{d}$ systems using and NRQCD $b$ quark and an improved staggered light quark. This calculation is complicated by the fact that the fitted correlators include an oscillatory contribution from the parity partner of the desired meson. This is a consequence of using staggered light quarks.

The results we present here are preliminary, and the reader is referred to [10] for a more complete treatment. In figure 5 we plot the quantity $2 m_{B_{s}}-m_{\Upsilon}$. Terms explicit in the quark mass cancel in this difference, exposing the differences in the binding energies of the systems. Our determination of $2 m_{B_{s}}-m_{\Upsilon}$ seems to be systematically too large, but we present here only a statistical error and delay a systematic determination of the errors until [10], where further results will also be presented. It should however be noted that the sea $s$ quark mass included was estimated to be to high by approximately $30 \%$.

As yet our results for the $B_{s}$ and $B_{d}$ do not support any conclusions, but calculations increasing the number of light quark masses and simulating the $D_{s}$ and $D$ with NRQCD $c$ quarks are currently being analysed. Given the success of our heavy quarkonium calculations, there is a good prospect that these calculations will yield useful results. 


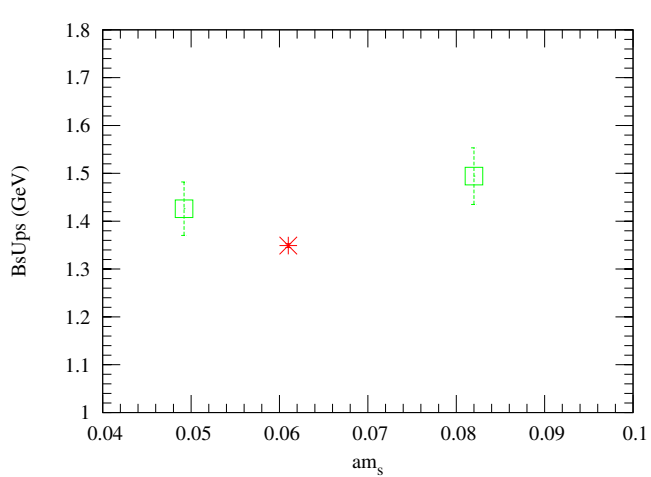

Figure 5: $2 m_{B_{s}}-m_{v}$. The boxes are our results and the star is experimental value placed at the physical $s$ quark mass $\left(a m_{s}=0.062\right)$ for this ensemble.

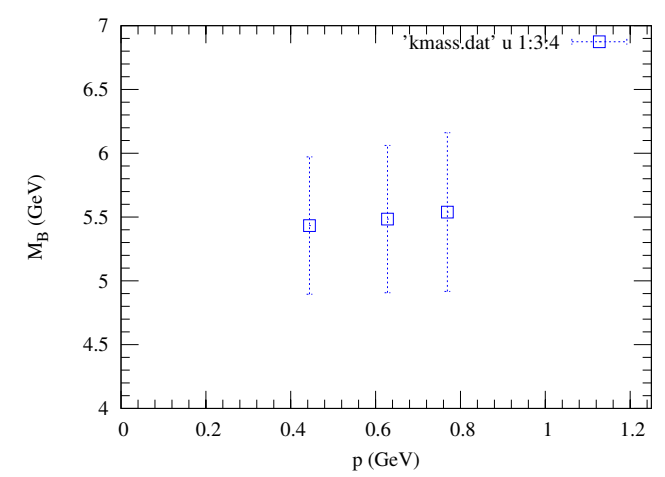

Figure 6: The Kinetic mass of $B_{s}$ in physical units vs. spatial momenta. The errors shown are purely statistical. Experimentally $M_{B_{s}}=$ 5.3696(24) GeV

We thank James Simone and the Fermilab lattice group for the use of their light quark propagators and computer time at the Fermilab cluster. Fermilab is operated by the Universities Research Association Inc. under contract with the US Department of Energy. I.A and C.D are supported by the UK Particle Physics and Astronomy Research Council. A.G. was supported by the US Department of Energy.

\section{References}

[1] G. P. Lepage, L. Magnea, C. Nakhleh, U. Magnea and K. Hornbostel, Improved nonrelativistic qcd for heavy quark physics, Phys. Rev. D46 (1992) 4052-4067 [hep-lat/9205007].

[2] C. W. Bernard et. al., The qcd spectrum with three quark flavors, Phys. Rev. D64 (2001) 054506 [hep-lat/0104002].

[3] C. Quigg and J. L. Rosner, Quantum mechanics with applications to quarkonium, Phys. Rept. 56 (1979) 167-235.

[4] G. P. Lepage et. al., Constrained curve fitting, Nucl. Phys. Proc. Suppl. 106 (2002) 12-20 [hep-lat/0110175].

[5] A. Gray et. al., The upsilon spectrum and $m(b)$ from full lattice qcd, hep-lat/0507013.

[6] CP-PACS Collaboration, Y. Kayaba et. al., Charmed meson spectra and decay constants with one-loop o(a) improved relativistic heavy quark action, hep-lat/0409086.

[7] HPQCD Collaborarion Collaboration, I. F. Allison et. al., A precise determination of the b/c mass from dynamical lattice qcd, Nucl. Phys. Proc. Suppl. 140 (2005) 440-442 hep-lat/0 09090.

[8] HPQCD Collaboration, I. F. Allison et. al., Mass of the b/c meson in three-flavor lattice qcd, Phys. Rev. Lett. 94 (2005) 172001 hep-lat/0411027.

[9] CDF Collaboration, M. D. Corcoran, Recent results on the b/c meson, hep-ex/0506061.

[10] I.Allison, b and c physics in lattice qcd with light sea quarks. in preparation, . 